\title{
Emerging Role of Mass Spectrometry on Clinical Setting: Critical Issues and Perspectives
}

\author{
Alessio Cortelazzo* \\ Department of Medical Biotechnologies, University of Siena, Italy
}

Submission: May 21, 2018; Published: May 29, 2018

*Corresponding author: Alessio Cortelazzo, Department of Medical Biotechnologies, University of Siena, Siena, Italy, Email: corteale@gmail.com

\section{Before Mass Spectrometry Finds Application in the Real Clinical Setting}

Before the latter half of the 20th century, no translation into routine clinical practice of several molecules was achieved during MS-based biochemical research applications. Criticism was raised, especially regarding the reproducibility of the MS studies performed. The most widely criticized items in MS-based biomarker discovery studies were

a) The adoption of standardized operating procedures for pre-analytical and analytical items,

b) Poor study design,

c) Lack of multi-centric studies, and

d) Unreliable data evaluation and statistical analyses [1].

The lack of standardization, widely considered a relevant source of random and systematic errors, has been the basis for uncertainty of analytical results and poor comparability. The successful use of mass spectrometry for the discovery of clinically-relevant novel possible molecular biomarkers required a careful consideration of standardized operating procedures, for pre-analytical variables, including samples handling and storage [1].

Primary Limitations of Mass Spectrometry and Challenges

MS applications associated with gas chromatography separation (GC-MS) have been the "gold standard" in specialized clinical laboratories for the quantitation of drugs, organic acids and steroids. A primary limitation of GC-MS was that analytes needed to be volatile and thus most clinical assays required multiple extraction/purification steps along with a chemical derivatization to render the analytes sufficiently volatile for analysis [2].

Yet, there are still problems in the clinical application of MS, including sample preparation, online extraction, throughput, automation, laboratory information system interfacing, interinstruments standardization and harmonization, and Food and Drug Administration (FDA) regulation.

The widespread implementation of expanded newborn screening programs using tandem MS "profiling" of amino acids and acyl-L-carnitines has been effective and beneficial. Over time, quantitative analyzes are required. Ms "profiles" are not quantitative: neither standardized calibrants nor multiple-point calibration curves are used.

MS is slowly transforming the practice of laboratory medicine and is being driven by several factors, including improved analytical specificity and sensitivity. The measurement of thyroglobulin is a clinical example for which MS has been shown to offer superior assay quality compared to traditional immunoassays.

The main challenges for MS include

a) The high capital cost of equipment,

b) Requirements for a skilled labor force,

c) Lack of automation, and

d) Regulatory uncertainty [3].

i. Cost reduction is considered a major pressure driving the adoption of MS but the initial capital cost of the equipment is high and laboratory expertise in the development, validation, and maintenance of MS-based assays may be limited.

ii. The requirement for skilled labor is especially acute for MS applications based on laboratory-developed challenges tests (LDTs). LDTs require considerable expertise in method development and validation. These talents are difficult to acquire in a training environment and generally require several years of practical experience in a functional MS laboratory to become proficient. 
iii. Lack of automation for both sample preparation and data reporting and (FDA) regulations on laboratorydeveloped tests are great examples that require multiple parties to work together to come up with better solutions for the field.

iv. The area of LDTs has also been evolving in recent years as the FDA and other related agencies determine how best to regulate an area that has been somewhat of a grey area.

The FDA's "Analytical Procedures and Methods Validation for Drugs and Biologics: Guidance for Industry", along with other such guidelines, defines approaches that are proven to provide excellent accuracy and precision when quantifying analytes in biological matrices.

\section{Today, MS Continues to Adapt and Expand into New Clinical Applications}

For capacity, accuracy, and specificity of analysis, the use of MS in clinical diagnostics and clinical research has grown dramatically in recent years. Clinical applications of MS continue to expand, and MS is now being used in almost all areas of laboratory medicine.

Various types of MS with high specificity (i.e., liquid chromatography coupled with MS; matrix-assisted laser desorption/ionization/time-of-flight-MS, MALDI-TOF-MS), are being increasingly valued and utilized as tools in clinical laboratories. These MS techniques overcome the limitations of immunoassays and offer many advantages over earlier approaches [4].

A particularly effective tool for improving throughput of MS has been the development of multiplex assays. Nowadays, main novel applications of MS include clinical imaging applications and microbiology. The use of MS for microbiology testing is a somewhat distinct application which appears poised for explosive growth over the next several years. The major advantages over alternatives are its ability to identify microbes in a much shorter time, with less work, and with greater accuracy. From a culture or colony, the system can identify the microbes in minutes rather than many hours required with older techniques. In a clinical diagnostic setting, the time savings can equate to a major benefit in terms of treatment and economics [5].

\section{At the Near Future MS-Based Laboratory: Critical Points and Perspectives}

MS is unsurpassed in its combination of sensitivity, specificity, and speed. Fully computerized and reliable instruments have made possible the use of this powerful tool in clinical medicine. While MS has grown rapidly and holds great promise, remain several critical-points relating to its use in clinical applications, including its complexity, high upfront cost, lack of userfriendliness, low throughput as well as the complexity of the science and the associated regulatory process [6].

A fundamental step for setting up an MS-based laboratory service is deciding what instrumentation to purchase. The choice of instrumentation mainly depends on the analytes of interest. For most small-molecule quantitative methods (i.e., testosterone, vitamin D and drugs of abuse) a triple-quadrupole MS with LC is the instrument of choice. Triple-quadrupole mass spectrometers are also used for newborn screening and for quantification of peptides and proteins. For a clinical microbiology laboratory interested in qualitative identification of a variety of microbes, a MALDI-TOF-MS is optimal [6].

In the next future, with emerging technologies in MS, we expect to see more robust and reliable MS applications with a broad menu of tests that will become routine diagnostic tools in clinical laboratories.

As MS moves deeper into the clinical space, some vendors are offering "platforms certified" for clinical use, which means handling regulatory requirements.

The miniaturization of MS systems being pursued at places and could allow a transportable device that minimizes the specialized skill set required for operators and allows for rapid and accurate MS analysis in a point-of-care format.

\section{References}

1. Himmelsbach M (2012) 10 years of MS instrumental developments-impact on LC-MS/MS in clinical chemistry. J Chromatogr B Analyt Technol Biomed Life Sci 883-884: 3-17.

2. Dorman FL, Whiting JJ, Cochran JW, Torresdey JG (2010) Gas chromatography. Anal Chem 82(12): 4775-4785.

3. Vaysse PM, Heeren RMA, Porta T, Balluff B (2017) Mass spectrometry imaging for clinical research - latest developments, applications, and current limitations. Analyst 142(15): 2690-2712.

4. Strathmann FG, Hoofnagle AN (2011) Current and future applications of mass spectrometry to the clinical laboratory. Am J Clin Pathol 136(4): 609-616.

5. Sloan A, Wang G, Cheng K (2017) Traditional approaches versus mass spectrometry in bacterial identification and typing. Clin Chim Acta 473: $180-185$

6. Jannetto PJ Fitzgerald RL (2016) Effective Use of Mass Spectrometry in the Clinical Laboratory. Clin Chem 62(1): 92-98. 
This work is licensed under Creative Commons Attribution 4.0 License

DOI: 10.19080/JOJCS.2018.07.555705

\section{Your next submission with Juniper Publishers} will reach you the below assets

- Quality Editorial service

- Swift Peer Review

- Reprints availability

- E-prints Service

- Manuscript Podcast for convenient understanding

- Global attainment for your research

- Manuscript accessibility in different formats ( Pdf, E-pub, Full Text, Audio)

- Unceasing customer service

Track the below URL for one-step submission https://juniperpublishers.com/online-submission.php 